\title{
Analytic Expressions for the Isosteric Heat of Adsorption from Adsorption Isotherm Models and 2D SAFT-VR EoS
}

\author{
Flor Siperstein ${ }^{1}$, Carlos Avendaño ${ }^{1}$, Jordan Ortiz ${ }^{2}$, and Alejandro Gil-Villegas ${ }^{2}$ \\ ${ }^{1}$ The University of Manchester \\ ${ }^{2}$ Universidad de Guanajuato
}

June 2, 2020

\begin{abstract}
The isosteric heat of adsorption is an important thermodynamic property used to characterise and optimise adsorption processes. In this work, analytic expressions for isosteric heats of adsorption are derived for a collection of commonly used isotherm models and a two-dimensional molecular equation of state based on the SAFT-VR approach. The use of these expressions is presented with an example of adsorption of nitrous oxide, $\mathrm{N}_{2} \mathrm{O}$, on biochar, which is a waste biomass charcoal that exhibits high adsorption potential. The results show that accurate fitting of the adsorption isotherms leads to consistent results obtained with different approaches, however, the predicted isosteric heat of adsorption exhibits strong variations in the regions where experimental data is insufficient such in the region of low pressure/low coverage. Convergence on the prediction of the isosteric heat of adsorption by the different models is only observed in the region where no extrapolation of experimental data is needed.
\end{abstract}

\section{Introduction}

Adsorption is a ubiquitous separation technique in science and engineering and, in many cases, is preferred over other alternatives for separation of gases due to its low economic cost compared to separation techniques such as cryogenic distillation and membrane separation that requires low temperatures conditions and pumping at high pressures, respectively (Nicholson \& Gubbins, 1996). Theoretical understanding of adsorption is challenging due to the many factors that affect the process such as roughness of the adsorbent, pore size and shape distributions, as well as local fluid-fluid and fluid-solid interactions (Balbuena \& Gubbins, 1993). The isosteric heat of adsorption, also known as differential enthalpy of adsorption, is a valuable thermodynamic property used for the design and optimisation of adsorption processes as it provides information about adsorbate-adsorbate and adsorbate-adsorbent interactions. In homogeneous adsorbents, for example, the isosteric heat of adsorption remains constant at low adsorbate loadings indicating that the interactions between adsorbed molecules do not contribute to the adsorption energy. At high adsorbate loading, however, lateral adsorbate-adsorbent interactions become significant, thus causing an increase on the isosteric heat of adsorption (Snurr et al., 1993; Sircar \& Cao, 2002). A different behaviour is observed in energetically heterogeneous adsorbents in which the isosteric heat of adsorption decreases as the adsorbate loading increases as molecules tend to adsorb on high-energy sites even at low pressures (Sircar \& Cao, 2002).

The isosteric heat of adsorption for pure components can be obtained from calorimetric measurements or from adsorption isotherms measured at different temperatures. Under this approach, a Clapeyron-type 
relationship is used to determine the isosteric heat of adsorption $Q_{\mathrm{st}}$ :

$$
Q_{\mathrm{st}}=R T^{2}\left(\frac{\partial \ln p}{\partial T}\right)_{n}
$$

where $n$ is the amount adsorbed, $p$ and $T$ are the pressure and absolute temperature of the system, and $R$ is the universal gas constant. Details for the derivation of Equation (1) can be found elsewhere (Hill, 1949). Equation (1) is only valid for pure components and perfect gases and has lead to some confusion when working with mixtures or high pressure systems. Therefore, Myers (Myers, 2002) suggested to use the concept of differential enthalpy of adsorption, $\Delta \bar{h}_{i}^{a}$, defined as the partial molar enthalpy of adsorption:

$$
\Delta \bar{h}_{i}^{a}=\left[\frac{\partial \Delta H^{a}}{\partial n_{i}^{a}}\right]_{T, n_{j}^{a}}=\bar{h}_{i}^{a}-h_{i}^{\circ}=-R T^{2}\left[\frac{\partial \ln f_{i}}{\partial T}\right]_{n_{i}^{a}, n_{j}^{a}}
$$

where $\Delta H^{a}$ is the integral enthalpy of adsorption, $\bar{h}_{i}^{a}$ and $h_{i}^{\circ}$ are the molar enthalpies of the adsorbed phase and in the perfect gas reference state; $f_{i}$ is the fugacity of component $i$, and $n_{i}^{a}$ is the amount adsorbed of component $i$. Other authors have also used the concept of partial molar enthalpies of adsorption to study adsorption of liquids and mixtures (Builes et al., 2013).

$Q_{\text {st }}$ and $\Delta \bar{h}_{i}^{a}$ are the same for pure components at low pressures (where the ideal gas approximation is valid). Analytic expressions for $Q_{\text {st }}$ or $\Delta \bar{h}^{a}$ are readily available for pure components using simple isotherm models or 2-dimensional (2D) fluids (Hill, 1949), but not necessarily for those that accurately represent experimental data. In this paper we derive a collection of expressions that can be used to determine $Q_{\mathrm{st}}$, or equivalent $\Delta \bar{h}^{a}$, for a variety of commonly used adsorption isotherms: extensions of the Langmuir model (Langmuir, 1916; Honig \& Mueller, 1962; Nitta et al., 1984; Martinez \& Basmadjian, 1996; Yao, 2000; Bai \& Yang, 2001; Zhang \& Wang, 2010), virial-type adsorption isotherms (Czepirski \& Jagiello, 1989; Siperstein \& Myers, 2001), Freundlich (Freundlich, 1906), Sips (Sips, 1948) and Toth (Toth, 1971) isotherms, as well as a two-dimensional (2D) equation of state (EoS) based on perturbation theory, within the SAFT-VR approach framework (Gil-Villegas et al., 1997; Martínez et al., 2007; Jiménez-Serratos et al., 2008; Trejos et al., 2014). As an example, we apply the derived expressions to the adsorption of $\mathrm{N}_{2} \mathrm{O}$ on biochar to compare the results obtained from using common isotherms and the molecular-based 2D-SAFT-VR EoS.

\section{Adsorption Models}

\section{Langmuir model}

Equation (1) is easily applied when the adsorption isotherm is written with the amount adsorbed as independent variable, and the pressure as calculated variable. Take as an example the Langmuir adsorption isotherm given by

$$
n=\frac{n_{\mathrm{sat}} b p}{1+b p}
$$


where $n$ is the amount adsorbed, $n_{\text {sat }}$ is the saturation capacity, $b$ is the adsorption affinity, and $p$ is the pressure of the system. The Langmuir isotherm can also be written as:

$$
p=\frac{1}{b}\left(\frac{n / n_{\mathrm{sat}}}{1-n / n_{\mathrm{sat}}}\right) .
$$

Following the assumptions of the Langmuir model where the saturation capacity $n_{\text {sat }}$ is independent of temperature, but the adsorption affinity $b$ depends on temperature, the isosteric heat of adsorption for the Langmuir model can be written as:

$$
Q_{\mathrm{st}}=R T^{2}\left(\frac{\partial \ln p}{\partial T}\right)_{n}=-R T^{2}\left(\frac{d \ln b}{d T}\right)
$$

Various temperature dependences have been suggested for the adsorption affinity. Assuming that the temperature dependence of the adsorption affinity follows an Arrhenius expression (Myers, 2002):

$$
b=b_{0} \exp \left(\frac{-E}{R T}\right)
$$

then

$$
\frac{d \ln b}{d T}=\frac{d \ln b_{0}}{d T}+\frac{d}{d T}\left(\frac{-E}{R T}\right)=\frac{E}{R T^{2}}
$$

where the pre-exponential factor $b_{0}$ is related to the entropy of saturation, and $E$ to the differential enthalpy of adsorption, where it is understood that $E<0$. Therefore, the isosteric heat of adsorption for the Langmuir model is simply given by

$$
Q_{\mathrm{st}}=-E,
$$


assuming that $b_{0}$ is independent of temperature. Formally, the pre-exponential factor $b_{0}$ in the adsorption affinity is a function of temperature (Hill, 1949; Lee, 1988), and is related to the vibrational partition function of the adsorbed molecule and the ideal gas contribution. In the classical limit, the temperature dependence of the pre-exponential factor can be written as:

$$
\begin{aligned}
b & =\left[\left(\frac{h^{2}}{2 \pi m k T}\right)^{3 / 2} \frac{1}{k T}\left(\frac{k T}{h \nu}\right)^{3}\right] \exp \left(\frac{-E^{\prime}}{R T}\right) \\
& =b_{0}^{\prime} T^{1 / 2} \exp \left(\frac{-E^{\prime}}{R T}\right)
\end{aligned}
$$

where $h$ is the Planck's constant, $k$ is the Boltzmann constant, $m$ is the mass of the particle, and $\nu$ is the frequency of vibration of atoms adsorbed on the substrate, therefore

$$
\frac{d \ln b}{d T}=\frac{d \ln b_{0}^{\prime}}{d T}+\frac{1}{2} \frac{d \ln T}{d T}+\frac{d}{d T}\left(\frac{-E^{\prime}}{R T}\right)=\frac{1}{2 T}+\frac{E^{\prime}}{R T^{2}},
$$

and the resulting isosteric heat can be written as:

$$
Q_{\mathrm{st}}=-E^{\prime}-\frac{1}{2} R T .
$$

The Langmuir model assumes that the saturation capacity $n_{\text {sat }}$ is independent of temperature, but adsorption may not take place on well defined sites in practical applications and as a consequence there is a temperature dependence of the saturation capacity as suggested by Hill (Hill, 1949). Then the isosteric heat of adsorption for the Langmuir model with temperature dependent $n_{\text {sat }}$ is be given by

$$
\begin{aligned}
Q_{\mathrm{st}} & =-R T^{2}\left[\frac{d \ln b}{d T}+\frac{d \ln \left(n_{\mathrm{sat}}-n\right)}{d T}\right] \\
& =-R T^{2} \frac{d \ln b}{d T}-\frac{R T^{2}}{n_{\mathrm{sat}}-n} \frac{d n_{\mathrm{sat}}}{d T}
\end{aligned}
$$


A functional form for the temperature dependence of the saturation capacity can be given by

$$
\Gamma=-\frac{d \ln n_{\mathrm{sat}}}{d T}=-\frac{1}{n_{\mathrm{sat}}} \frac{d n_{\mathrm{sat}}}{d T},
$$

where $\Gamma$ is a measure of the adsorbed phase thermal expansion coefficient. Therefore, the expression for the isosteric heat of adsorption for the modified Langmuir isotherm that follows is:

$$
\begin{aligned}
Q_{\mathrm{st}} & =R T^{2}\left(\frac{\partial \ln p}{\partial T}\right)_{n}=-R T^{2}\left[\frac{d \ln b}{d T}-\frac{n_{\mathrm{sat}}}{n_{\mathrm{sat}}-n} \Gamma\right] \\
& =-R T^{2}\left[\frac{d \ln b}{d T}-(1+b p) \Gamma\right]
\end{aligned}
$$

\section{Multisite Langmuir model}

An analytic expression for the isosteric heat of adsorption is not necessarily straight forward when the adsorption isotherms become more complex. An expression for the isosteric heat of adsorption for the dualsite Langmuir model has been reported (missing citation), where an explicit expression for the pressure $p$ as a function of the amount adsorbed $n$ is obtained from a quadratic equation and used in Equation (1). Starting from the dual-site Langmuir equation:

$$
n=\frac{n_{\mathrm{sat}, 1} b_{1} p}{1+b_{1} p}+\frac{n_{\mathrm{sat}, 2} b_{2} p}{1+b_{2} p},
$$

the pressure is given as:

$$
p=\frac{\left(\beta^{2}+4 \alpha n\right)^{0.5}-\beta}{2 \alpha}
$$


where:

$$
\alpha=\left(n_{\mathrm{sat}, 1}+n_{\mathrm{sat}, 2}-n\right) b_{1} b_{2},
$$

and

$$
\beta=\left(n_{\mathrm{sat}, 1}-n\right) b_{1}+\left(n_{\mathrm{sat}, 2}-n\right) b_{2}
$$

The expression for calculating the isosteric heat of adsorption assuming that the number of sites is independent of temperature is given by:

where

$$
\frac{d \alpha}{d T}=\left(n_{\mathrm{sat}, 1}+n_{\mathrm{sat}, 2}-n\right)\left(b_{1} \frac{b_{2}}{d T}+b_{2} \frac{d b_{1}}{d T}\right)
$$

and

$$
\frac{d \beta}{d T}=\left(n_{\mathrm{sat}, 1}-n\right)\left(\frac{d b_{1}}{d T}\right)+\left(n_{\mathrm{sat}, 2}-n\right)\left(\frac{d b_{2}}{d T}\right)
$$

An alternative approach to obtain the isosteric heat of adsorption is to differentiate implicitly any adsorption isotherm where the pressure appears as independent variable. For the dual-site Langmuir model in Equation (15) with the number of sites independent of temperature we have:

$$
\left(\frac{\partial n}{\partial T}\right)_{n}=\left(\frac{\partial}{\partial T}\left(\frac{n_{\mathrm{sat}, 1} b_{1} p}{1+b_{1} p}+\frac{n_{\mathrm{sat}, 2} b_{2} p}{1+b_{2} p}\right)\right)_{n}=0
$$


that leads to:

$$
\left(\frac{\partial p}{\partial T}\right)_{n}=-\frac{n_{\mathrm{sat}, 1}\left(1+b_{2} p\right)^{2} p\left(\frac{d b_{1}}{d T}\right)+n_{\mathrm{sat}, 2}\left(1+b_{1} p\right)^{2} p\left(\frac{d b_{2}}{d T}\right)}{n_{\mathrm{sat}, 1} b_{1}\left(1+b_{2} p\right)^{2}+n_{\mathrm{sat}, 2} b_{2}\left(1+b_{1} p\right)^{2}}
$$

resulting in an expression for the isosteric heat of adsorption as:

$$
Q_{\mathrm{st}}=-R T^{2} \frac{n_{\mathrm{sat}, 1}\left(1+b_{2} p\right)^{2}\left(\frac{d b_{1}}{d T}\right)+n_{\mathrm{sat}, 2}\left(1+b_{1} p\right)^{2}\left(\frac{d b_{2}}{d T}\right)}{n_{\mathrm{sat}, 1} b_{1}\left(1+b_{2} p\right)^{2}+n_{\mathrm{sat}, 2} b_{2}\left(1+b_{1} p\right)^{2}}
$$

This approach is easily generalised to a multisite Langmuir isotherm, with $i$ number different types of adsorption sites (Nitta et al., 1984):

$$
n=\sum_{i} \frac{n_{\mathrm{sat}, \mathrm{i}} b_{i} p}{1+b_{i} p}
$$

where

$$
\left(\frac{\partial p}{\partial T}\right)_{n}=-\left[\sum_{i} \frac{n_{\mathrm{sat}, i} b_{i} p}{\left(1+b_{i} p\right)^{2}} \frac{d \ln b_{i}}{d T}\right]\left[\sum_{i} \frac{n_{\mathrm{sat}, i} b_{i}}{\left(1+b_{i} p\right)^{2}}\right]^{-1}
$$

It is easy to show that Equation (25) reduces to Equation (22) for the case when only two types of sites are present. Then the isosteric heat of adsorption for the multisite-Langmuir model with number of sites independent of temperature is given by:

$$
Q_{\mathrm{st}}=-R T^{2}\left[\sum_{i} \frac{n_{\mathrm{sat}, i} b_{i}}{\left(1+b_{i} p\right)^{2}} \frac{d \ln b_{i}}{d T}\right]\left[\sum_{i} \frac{n_{\mathrm{sat}, i} b_{i}}{\left(1+b_{i} p\right)^{2}}\right]^{-1}
$$


A more general case could also consider that the number of sites or maximum amount adsorbed depends on temperature, as shown in Equation (13). Then, the isosteric heat of adsorption becomes:

$$
Q_{\mathrm{st}}=-R T^{2} \frac{\left[\sum_{i} \frac{n_{\mathrm{sat}, \mathrm{i}} b_{i}}{\left(1+b_{i} p\right)^{2}} \frac{d \ln b_{i}}{d T}+\sum_{i} \frac{b_{i} n_{\mathrm{sat}, \mathrm{i}}}{\left(1+b_{i} p\right)} \frac{d \ln n_{\mathrm{sat}, \mathrm{i}}}{d T}\right]}{\left[\sum_{i} \frac{n_{\mathrm{sat}, \mathrm{i}} b_{i}}{\left(1+b_{i} p\right)^{2}}\right]}
$$

or in terms of the adsorbed phase expansion coefficient:

$$
Q_{\mathrm{st}}=-R T^{2} \frac{\left[\sum_{i} \frac{n_{\mathrm{sat}, \mathrm{i}} b_{i}}{\left(1+b_{i} p\right)^{2}} \frac{d \ln b_{i}}{d T}-\sum_{i} \frac{b_{i} n_{\mathrm{sat}, \mathrm{i}} \Gamma_{i}}{\left(1+b_{i} p\right)}\right]}{\left[\sum_{i} \frac{n_{\mathrm{sat}, \mathrm{i}} b_{i}}{\left(1+b_{i} p\right)^{2}}\right]} .
$$

A generalisation of the Langmuir model (Martinez \& Basmadjian, 1996) captures the variation in the asymptotic amount adsorbed at different temperatures by including the exponents $s$ and $t$ in the following equation:

$$
p=\frac{1}{b} \exp \left(-\frac{\eta u N_{0} n / n_{\text {sat }}}{R T}\right) \times\left(\frac{\left(n / n_{\text {sat }}\right)^{s}}{\left(1-n / n_{\text {sat }}\right)^{t}}\right)
$$

where $u, \eta$, and $n_{\text {sat }}$ are constants and $b$ has the temperature dependence given in Equation (6). Then the isosteric heat of adsorption is given by:

$$
Q_{\mathrm{st}}=-E-\eta u N_{0} n / n_{\mathrm{sat}},
$$

assuming that both $s$ and $t$ are independent of temperature. 


\section{Virial equation}

The virial equation to describe adsorption of fluids in porous materials can be written as a polynomial expansion of the form (Czepirski \& Jagiello, 1989):

$$
\ln p=\ln n+\sum_{0}^{i} c_{i} n^{i},
$$

where the Henry's constant $b$ is given as $\ln b=-c_{0}$ and the temperature dependence for the other constants has been suggested to have the following form:

$$
c_{i}=\frac{a_{i}}{T}+b_{i},
$$

where $a_{i}$ and $b_{i}$ are adjustable parameters.

For this isotherm, the isosteric heat of adsorption is given as:

$$
Q_{\mathrm{st}}=-R \sum_{0}^{i} a_{i} n^{i}
$$

The virial isotherm given in Equation (31) is unable to describe adsorption isotherms with well defined saturation capacities. An alternative formulation using a Langmuir type saturation capacity is sometimes used (Siperstein \& Myers, 2001):

$$
\ln p=\ln \frac{n}{n_{\mathrm{sat}}-n}+\sum_{0}^{i} c_{i} n^{i}
$$

where the isosteric heat of adsorption is given by:

$$
Q_{\mathrm{st}}=-R T^{2} \Gamma \frac{n_{\mathrm{sat}}}{n_{\mathrm{sat}}-n}-R \sum_{0}^{i} a_{i} n^{i}
$$




\section{Freundlich and Sips models}

Freundlich proposed an simple and empirical model with two adjustable parameters that is often used to describe adsorption of organic vapours on activated carbon and other heterogeneous surfaces, i.e. materials with different types of adsorption sites with different energies of adsorption (Freundlich, 1906). The Freundlich isotherm is given by

$$
n=b p^{t}
$$

where $b$ is the Freundlich constant that denotes that adsorption capacity of the substrate and $t$ is an empirical exponent that indicates the adsorption intensity of the substrate.

The isosteric heat of adsorption for this isotherm, assuming that both adjustable parameters are temperature dependent is given as:

$$
Q_{\mathrm{st}}=-\frac{R T^{2}}{t}\left(\frac{d \ln b}{d T}+\ln p \frac{d t}{d T}\right)
$$

An extension of the Freundlich model, proposed by Sips (Sips, 1948), is often referred to as the LangmuirFreundlich model, where the adsorption isotherm is given by:

$$
n=\frac{n_{\text {sat }}(b p)^{t}}{1+(b p)^{t}},
$$

and the isosteric heats of adsorption assuming that all adjustable parameters are temperature dependent is given by:

$$
Q_{\mathrm{st}}=-R T^{2}\left[\frac{d \ln b}{d T}+\frac{1+(b p)^{t}}{t} \frac{d \ln n_{\mathrm{sat}}}{d T}+\frac{\ln (b p)}{t} \frac{d t}{d T}\right]
$$




\section{Toth}

The Freundlich and Sips adsorption isotherm models can describe well experimental data but they have an important disadvantage: the slope of the adsorption isotherm at the limit of zero pressure is not defined. The isotherm model proposed by Toth (Toth, 1971) overcomes this problem and is given by

$$
n=\frac{n_{\mathrm{sat}} b p}{\left[1+(b p)^{t}\right]^{1 / t}}
$$

where $n_{\text {sat }}, b$ and $t$ are adjustable parameters. The isosteric heat of adsorption for this model is given by:

\section{BET}

The BET equation (Brunauer et al., 1938) is often used for porous materials characterisation because it allows estimating the surface area of a mesoporous or macroporous material under certain assumptions. Even though the isotherms used with the BET model are often measured at a single temperature (77 K for nitrogen), if they were to be measured at different temperatures, it would be possible to estimate the isosteric heat of adsorption. Consider the BET isotherm written as:

$$
v=\frac{v_{\mathrm{m}} c p / p^{0}}{\left(1-p / p^{0}\right)\left[1+(c-1) p / p^{0}\right]}
$$

where $v$ is the volume adsorbed, $v_{\mathrm{m}}$ is the volume of the monolayer, $p$ is the pressure, $p^{0}$ is the saturation pressure at the temperature of the isotherm, and $c$ is an adjustable parameter, related to the difference in the energy of adsorption of the first layer and the other layers. Assuming that $v_{\mathrm{m}}$ and $c$ are independent of temperature, the isosteric heat of adsorption is given by:

$$
Q_{\mathrm{st}}=R T^{2} \frac{d \ln p^{0}}{d T}
$$

A more general expression can be obtained when considering that $v_{\mathrm{m}}$ and $c$ are temperature dependent, then the isosteric heat of adsorption is given as: 


$$
Q_{\mathrm{st}}=R T^{2}\left[\frac{d \ln p^{\circ}}{d T}-f_{1} \frac{d \ln c}{d T}-f_{2} \frac{d \ln v_{\mathrm{m}}}{d T}\right]
$$

where

$$
f_{1}=\frac{\left(1-p / p^{0}\right)^{2}}{1+(c-1)\left(p / p^{\circ}\right)^{2}}
$$

and

$$
f_{2}=\frac{\left(1-p / p^{0}\right)\left(1+(c-1) p / p^{0}\right)}{1+(c-1)\left(p / p^{\circ}\right)^{2}}
$$

\section{D-SAFT-VR equation of state}

Most adsorption models such as the Langmuir and the BET isotherms use the concept of adsorption sites in which equilibrium is described as the balance between the energy of the molecules adsorbed on these sticky sites and the gain of translational entropy from the desorption of the molecules from the substrate (Dill \& Bromberg, 2010). An alternative description of adsorption is introduced from the use of two-dimensional molecular-based equations of state where the adsorbed phase is represented as a 2D fluid in equilibrium with a 3D bulk phase (Machin \& Ross, 1962; Dash, 1975).

Within this approximation and the framework of the Barker and Henderson perturbation theory (Barker \& Henderson, 1967), it is possible to model the adsorption of particles interacting via a square-well (SW) pair potential (del Río \& Gil-Villegas, 1991). A more robust theoretical model for complex fluids comprised of non-linear associating molecules can be obtained through the use of more sophisticated molecular-based equations of state such as the Statistical Associating Fluid Theory originally developed by Gubbins and coworkers (Chapman et al., 1988; Jackson et al., 1988; Chapman et al., 1989; Chapman et al., 1990). Different incarnation of SAFT have been developed, but in this work we opt for the use of SAFT-VR methodology due to their flexibility in the shape of the pair intermolecular potential (Gil-Villegas et al., 1997; Galindo et al., 1998). Using this approach, Martinez et al.(Martínez et al., 2007) and Jimenez et al. (Jiménez-Serratos et al., 2008) reported the development of a 2D square-well SAFT-VR EoS for associating chain molecules. This theory has been applied in the modelling of adsorption on activated carbons, graphene and MOFs of several substances and their mixtures like nitrogen, methane, carbon dioxide, methanol, water and hydrogen (Castro et al., 2011; Trejos et al., 2014; Martínez et al., 2017), as well as complex fluids such as asphaltenes in porous rocks (Castro et al., 2009).

The 2D-SAFT-VR approach considers that bulk and adsorbed phases are formed by spherical particles of diameter $\sigma$ interacting via SW potentials with energy well depths and attractive ranges parameters 
$\left(\epsilon_{\text {bulk }}, \lambda_{\text {bulk }}\right)$ and $\left(\epsilon_{\text {ads }}, \lambda_{\text {ads }}\right)$ for both phases, respectively; interactions between bulk-adsorbed fluid particles particles are neglected whereas surface-adsorbed-particles interaction is also modelled with a SW potential with parameters $\left(\epsilon_{\mathrm{w}}, \lambda_{\mathrm{w}}\right)$. The expression for the isosteric heat of the 2D-SAFT-VR equation of state is given by:

$$
Q_{\mathrm{st}}=N_{0} \epsilon_{\mathrm{w}}+R T+4 \epsilon_{\mathrm{ads}} \gamma\left(\lambda_{\mathrm{ads}}^{2}-1\right) g_{\mathrm{hd}}\left(\sigma, \gamma_{\mathrm{eff}}\right) \Phi\left(\gamma_{\mathrm{eff}}\right)
$$

where $N_{0}$ is Avogadro's number, $\gamma$ and $g_{\text {hd }}$ are the $2 \mathrm{D}$ hard-disks packing fraction and radial distribution function, respectively, and $\gamma_{\text {eff }}$ and $\Phi$ are parameters of the mean-attractive energy of the adsorbed particles. Details of the theory and the derivation of Equation (41) are given in Appendix 1.

Table 1: Langmuir isotherm parameters, $n_{\text {sat }}$ and $b$, for the representation of the adsorption of $\mathrm{N}_{2} \mathrm{O}$ on biochar BC-60 at different temperatures reported by Cornelissen et al. (Cornelissen et al., 2013) The fitting error $\delta_{n}$ on the amount adsorbed $n$ is also reported.

\begin{tabular}{ccrc}
\hline$T / \mathrm{K}$ & $n_{\text {sat }} / \mathrm{cm}^{3} \mathrm{~g}^{-1}$ & $b / \mathrm{atm}^{-1}$ & $\delta_{n} \%$ \\
\hline 263.15 & 44.79 & 8.91 & 2.98 \\
273.15 & 40.20 & 7.09 & 2.93 \\
283.15 & 36.56 & 5.68 & 2.82 \\
293.15 & 33.31 & 4.28 & 2.54 \\
303.15 & 30.29 & 3.37 & 2.22 \\
313.15 & 27.59 & 2.58 & 1.95 \\
\hline
\end{tabular}




\section{Case of study: adsorption of $\mathrm{N}_{2} \mathrm{O}$ on biochar}

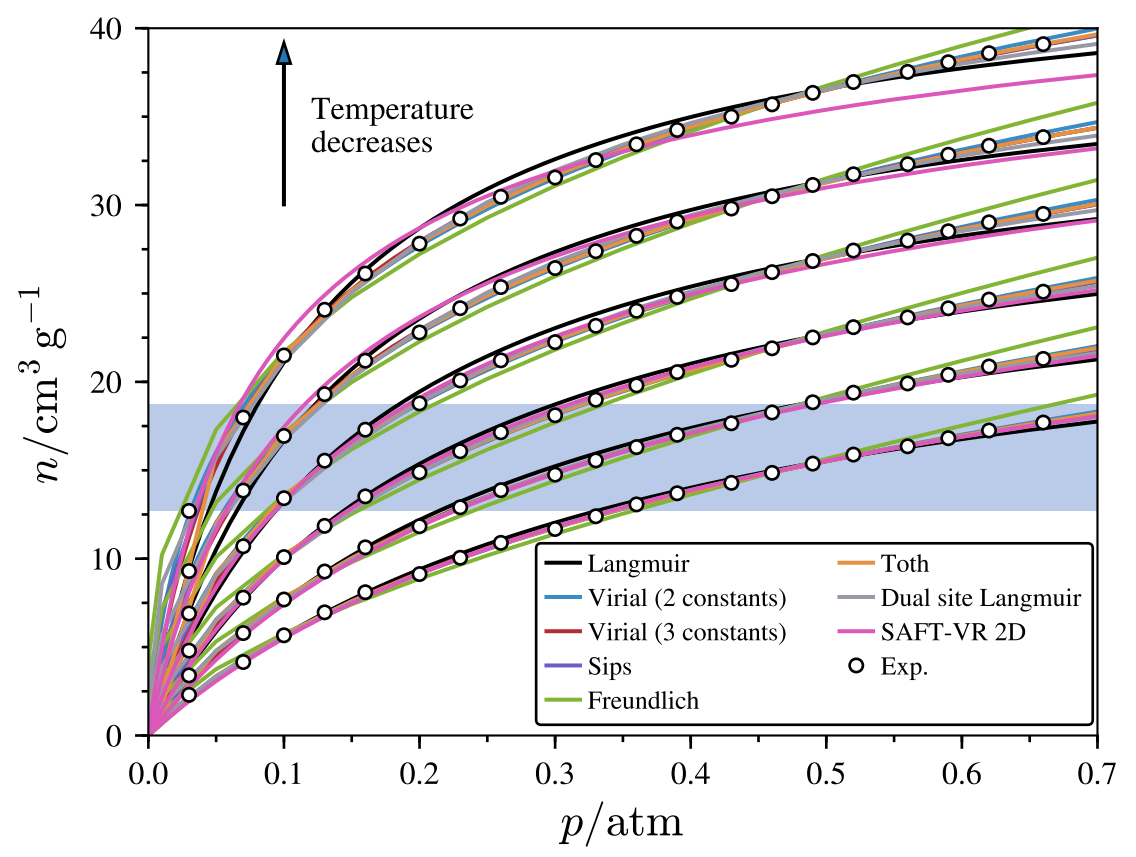

Figure 1: Adsorption isotherms for $\mathrm{N}_{2} \mathrm{O}$ on biochar BC-60 using experimental data from Cornelissen et al. (Cornelissen et al., 2013). The amount adsorbed $n$ is expressed as volume of $\mathrm{N}_{2} \mathrm{O}$ at STP per gram of sorbent, i.e., in units of $\mathrm{cm}^{3} \mathrm{~g}^{-1}$. From top to bottom, the temperatures correspond to the range $263.15 \mathrm{~K}$ to $313.15 \mathrm{~K}$ in increments of $10 \mathrm{~K}$, respectively. Symbols correspond to the experimental isotherms while solid curves are the fitted isotherms calculated using the different models introduced in Section. Parameters for these models are reported in Tables 2-9. The blue area indicates the region where at least one experimental point for each experimental isotherm is present. In this region, no extrapolation is needed to calculate the isosteric heats of adsorption.

Adsorption of $\mathrm{N}_{2} \mathrm{O}$ on biochar is taken as the case of study for the application of the various models to characterise adsorption isotherms and their associated equations for the isosteric heat of adsorptions derived in Section. Biochar is a waster carbon-rich porous material produced by thermal decomposition of biomass at low-oxygen conditions (Cornelissen et al., 2013; Li et al., 2017) that has has attracted considerably attention due to properties such as high stability against decay and high adsorption capacities of nutrients, which are properties attractive for soil enrichment, as well as their high capacity for adsorbing both heavymetals present in wastewater and green-house gases dispersed in the atmosphere (Lehmann, 2007). $\mathrm{N}_{2} \mathrm{O}$ is a powerful green-house gas that is known to have strong effects on the stratospheric ozone depletion. Despite the low contribution of $\mathrm{N}_{2} \mathrm{O}$ to total green-house gas emissions, its global potential warming is approximately 300 times more that $\mathrm{CO}_{2}$ (Thomson et al., 2012; Portmann et al., 2012).

We begin our discussion with the use of the Langmuir isotherm model to represent the experimental adsorption data of $\mathrm{N}_{2} \mathrm{O}$ on several types of biochars (BCs) and activated carbon (ACs) reported by Cornelissen et al. (Cornelissen et al., 2013). The values for the saturation capacity $n_{\text {sat }}$ and the adsorption affinity $b$ for the adsorption of $\mathrm{N}_{2} \mathrm{O}$ at different temperatures on a BC with a BET surface area of $60.1 \mathrm{~m}^{2} \mathrm{~g}^{-1}$, hereafter referred to as BC-60, are presented in Table 1 for pressures in the range $0.03 \mathrm{~atm}$ to $0.66 \mathrm{~atm}$. The values of these parameters are the same as those originally reported by Cornelissen et al. (Cornelissen et al., 2013). As expected, both $n_{\text {sat }}$ and $b$ decrease as the temperature decreases indicating a reduction of the adsorption 
Table 2: Temperature-dependent Langmuir isotherm parameters for different types of Biochars (BCs) and activated carbons (ACs). $E$ and $b_{0}$ correspond to the activation energy and pre-exponential factor in the Arrhenius-type model (c.f. Eq. (6)). $\Gamma$ corresponds to the adsorbed phase thermal expansion coefficient defined in Eq.(13). Experimental adsorption isotherms used for the fitting are taken from Cornelissen et al. (Cornelissen et al., 2013).

\begin{tabular}{lccc}
\hline Material & $E / \mathrm{kJ} \mathrm{mol}^{-1}$ & $b_{0} / \mathrm{atm}^{-1}$ & $\Gamma / \mathrm{K}^{-1}$ \\
\hline BC-60 & 17.0 & 0.00392 & 0.00961 \\
BC-176 & 15.8 & 0.00748 & 0.00904 \\
BC1 & 23.8 & 0.00016 & 0.01239 \\
BC-286 & 12.8 & 0.01128 & 0.00697 \\
AC-775 & 11.0 & 0.03567 & 0.01171 \\
AC-569 & 15.9 & 0.00484 & 0.00913 \\
\hline
\end{tabular}

Table 3: Parameters for the virial isotherm truncated to first order (2 constants) for the adsorption of $\mathrm{N}_{2} \mathrm{O}$ on BC-60. Experimental adsorption isotherms used for the fitting are taken from Cornelissen et al. (Cornelissen et al., 2013). The fitting error $\delta_{p}$ on the pressure $p$ is also reported. The gradients of the parameters $-\ln b$ and $c_{1}$ with respect to the temperature $T$ evaluated at $T=293.15 \mathrm{~K}$ are reported in the last row. The units of the parameter $b$ are $\left[\mathrm{cm}^{3} \mathrm{~g}^{-1} \mathrm{~atm}^{-1}\right]$.

\begin{tabular}{cccc}
\hline$T / \mathrm{K}$ & $-\ln (b)$ & $c_{1} / \mathrm{cm}^{-3} \mathrm{~g}$ & $\delta_{p} \%$ \\
\hline 263.15 & -6.925 & 0.0720 & 2.04 \\
273.15 & -6.318 & 0.0696 & 1.85 \\
283.15 & -5.807 & 0.0673 & 1.78 \\
293.15 & -5.259 & 0.0637 & 1.79 \\
303.15 & -4.828 & 0.0626 & 1.75 \\
313.15 & -4.386 & 0.0612 & 1.58 \\
\hline$\left.\frac{d}{d T}\right|_{T=293.15 \mathrm{~K}}$ & 0.0486 & -0.000216 & \\
\hline
\end{tabular}

Table 4: Parameters for the virial isotherm truncated to second order (3 constants) for the adsorption of $\mathrm{N}_{2} \mathrm{O}$ on BC-60. Experimental adsorption isotherms used for the fitting are taken from Cornelissen et al. (Cornelissen et al., 2013). The fitting error $\delta_{p}$ on the pressure $p$ is also reported. The gradients of the parameters $-\ln b, c_{1}$ and $c_{2}$ with respect to the temperature $T$ evaluated at $T=293.15 \mathrm{~K}$ are reported in the last row. The units of the parameter $b$ are $\left[\mathrm{cm}^{3} \mathrm{~g}^{-1} \mathrm{~atm}^{-1}\right]$.

\begin{tabular}{ccccc}
\hline$T / \mathrm{K}$ & $-\ln b$ & $c_{1} / \mathrm{cm}^{-3} \mathrm{~g}$ & $c_{2} / \mathrm{cm}^{-6} \mathrm{~g}^{2}$ & $\delta_{p} \%$ \\
\hline 263.15 & -6.368 & 0.032400 & $6.7 \times 10^{-4}$ & 2.10 \\
273.15 & -5.980 & 0.040469 & $5.9 \times 10^{-4}$ & 1.95 \\
283.15 & -5.593 & 0.045192 & $5.3 \times 10^{-4}$ & 1.93 \\
293.15 & -5.117 & 0.045349 & $5.4 \times 10^{-4}$ & 1.92 \\
303.15 & -4.727 & 0.046632 & $5.7 \times 10^{-4}$ & 1.87 \\
313.15 & -4.314 & 0.046714 & $6.4 \times 10^{-4}$ & 1.69 \\
\hline$\left.\frac{d}{d T}\right|_{T=293.15 \mathrm{~K}}$ & 0.0397 & 0.000254 & $2.0003 \times 10^{-6}$ & \\
\hline
\end{tabular}


Table 5: Parameters for the Freundlich isotherm for the adsorption of $\mathrm{N}_{2} \mathrm{O}$ on BC-60. Experimental adsorption isotherms used for the fitting are taken from Cornelissen et al. (Cornelissen et al., 2013). The fitting error $\delta_{n}$ on the amount adsorbed $n$ is also reported. The gradients of the parameters $b$ and $t$ with respect to the temperature $T$ evaluated at $T=293.15 \mathrm{~K}$ are reported in the last row.

\begin{tabular}{cccc}
\hline$T / \mathrm{K}$ & $b / \mathrm{cm}^{3} \mathrm{~g}^{-1} \mathrm{~atm}^{-t}$ & $t$ & $\delta_{n} \%$ \\
\hline 263.15 & 46.09 & 0.3271 & 2.55 \\
273.15 & 40.95 & 0.3782 & 2.86 \\
283.15 & 36.64 & 0.4305 & 3.09 \\
293.15 & 32.29 & 0.4988 & 3.35 \\
303.15 & 28.15 & 0.5560 & 3.60 \\
313.15 & 24.05 & 0.6199 & 3.78 \\
\hline$\left.\frac{d}{d T}\right|_{T=293.15 \mathrm{~K}}$ & -0.4371 & 0.4651 & \\
\hline
\end{tabular}

Table 6: Parameters for the Sips isotherm for the adsorption of $\mathrm{N}_{2} \mathrm{O}$ on BC-60. Experimental adsorption isotherms used for the fitting are taken from Cornelissen et al. (Cornelissen et al., 2013). The fitting error $\delta_{n}$ on the amount adsorbed $n$ is also reported. The gradients of the parameters $n_{\text {sat }}, b$ and $t$ with respect to the temperature $T$ evaluated at $T=293.15 \mathrm{~K}$ are reported in the last row.

\begin{tabular}{ccccc}
\hline$T / \mathrm{K}$ & $n_{\text {sat }} / \mathrm{cm}^{3} \mathrm{~g}^{-1}$ & $b / \mathrm{atm}^{-1}$ & $t$ & $\delta_{n} \%$ \\
\hline 263.15 & 62.16 & 3.560 & 0.6159 & 0.49 \\
273.15 & 56.20 & 2.830 & 0.6638 & 0.53 \\
283.15 & 51.66 & 2.277 & 0.7055 & 0.61 \\
293.15 & 46.83 & 1.843 & 0.7610 & 0.72 \\
303.15 & 41.48 & 1.626 & 0.8114 & 0.83 \\
313.15 & 36.24 & 1.434 & 0.8656 & 0.85 \\
\hline$\left.\frac{d}{d T}\right|_{T=293.15 \mathrm{~K}}$ & -0.5103 & -0.0419 & 0.00499 & \\
\hline
\end{tabular}


Table 7: Parameters for the Toth isotherm for the adsorption of $\mathrm{N}_{2} \mathrm{O}$ on BC-60. Experimental adsorption isotherms used for the fitting are taken from Cornelissen et al. (Cornelissen et al., 2013). The fitting error $\delta_{n}$ on the amount adsorbed $n$ is also reported. The gradients of the parameters $n_{\text {sat }}, b$ and $t$ with respect to the temperature $T$ evaluated at $T=293.15 \mathrm{~K}$ are reported in the last row.

\begin{tabular}{ccccc}
\hline$T / \mathrm{K}$ & $n_{\text {sat }} / \mathrm{cm}^{3} \mathrm{~g}^{-1}$ & $b / \mathrm{atm}^{-1}$ & $t$ & $\delta_{n} \%$ \\
\hline 263.15 & 75.03 & 25.851 & 0.4137 & 0.038 \\
273.15 & 68.71 & 13.801 & 0.4470 & 0.035 \\
283.15 & 65.12 & 8.290 & 0.4697 & 0.036 \\
293.15 & 63.04 & 4.576 & 0.4969 & 0.037 \\
303.15 & 57.94 & 2.936 & 0.5341 & 0.032 \\
313.15 & 48.50 & 1.969 & 0.6123 & 0.023 \\
\hline$\left.\frac{d}{d T}\right|_{T=293.15 \mathrm{~K}}$ & -0.4772 & -0.4449 & 0.00395 & \\
\hline
\end{tabular}

Table 8: Parameters for the dual-site Langmuir isotherm for the adsorption of $\mathrm{N}_{2} \mathrm{O}$ on BC-60. Experimental adsorption isotherms used for the fitting are taken from Cornelissen et al. (Cornelissen et al., 2013). The fitting error $\delta_{n}$ on the amount adsorbed $n$ is also reported. The gradients of the parameters $n_{\mathrm{sat}, 1}, b_{1}, n_{\mathrm{sat}, 2}$, and $b_{2}$ with respect to the temperature $T$ evaluated at $T=293.15 \mathrm{~K}$ are reported in the last row. The units of the parameter $n_{\mathrm{sat}, 1}$ and $n_{\mathrm{sat}, 2}$ are $\left[\mathrm{cm}^{3} \mathrm{~g}^{-1}\right]$, while the units of the parameters $b_{1}$ and $b_{2}$ are $\left[\mathrm{atm}^{-1}\right]$.

\begin{tabular}{cccccc}
\hline$T / \mathrm{K}$ & $n_{\text {sat }, 1}$ & $b_{1}$ & $n_{\text {sat }, 2}$ & $b_{2}$ & $\delta_{n} \%$ \\
\hline 263.15 & 39.90 & 4.935 & 8.201 & 447.3 & 0.76 \\
273.15 & 37.87 & 4.274 & 5.572 & 286.6 & 0.77 \\
283.15 & 36.16 & 3.516 & 4.035 & 192.1 & 0.76 \\
293.15 & 34.46 & 2.867 & 2.437 & 181.0 & 0.74 \\
303.15 & 32.09 & 2.430 & 1.441 & 167.7 & 0.82 \\
313.15 & 29.70 & 1.989 & 0.746 & 153.9 & 0.85 \\
\hline$\left.\frac{d}{d T}\right|_{T=293.15 \mathrm{~K}}$ & -0.2002 & -0.0598 & -0.1254 & -5.242 & \\
\hline
\end{tabular}

capacity of the material. The Langmuir isotherms using the parameters reported in Table 1 are shown in Figure 1, where it is observed that the fitting of the model to the experimental adsorption data is good, particularly at high temperatures. The error $\delta_{X}$ on the fitting of property $X$ for all the isothermal models presented in this work is quantified using the average absolute deviation given by

$$
\delta_{X}=\frac{1}{N_{p}} \sum_{i=1}^{N_{p}}\left|\frac{X_{\mathrm{e}, i}-X_{\mathrm{c}, i}}{X_{\mathrm{e}, i}}\right|
$$

where $X_{\mathrm{e}}$ and $X_{\mathrm{c}}$ are the experimental and computed values of variable $X$, respectively, and $N_{p}$ is the number of experimental points. For the Langmuir isotherm, the error on the fitting of the amount adsorbed $\delta_{n}$ is lower than $3 \%$ for all temperatures reported in the range $263 \mathrm{~K}$ to $313 \mathrm{~K}$.

A more general form of representing experimental data for different systems and temperatures is by obtaining the dependence of the Langmuir parameters as function of temperature for a specific system. The affinity parameter, for example, can be represented using an Arrhenius-type model given in Equation (6), in which the pre-exponential factor $b_{0}$ as well as activation energy $E$ can be determined using experimental data at different temperatures. For the case of the saturation capacity $n_{s}$, the temperature dependence is obtained through the calculation of $\Gamma$ defined in Eq. (13). Using again the experimental data reported by Cornelissen 
et al. (Cornelissen et al., 2013) for different types of ACs and BCs, the fitted values for $b_{0}, E$, and $\Gamma$ are reported in Table 2.

The reported experimental data was also fitted with other models described in this work: first- and secondorder virial, Freundlich, Sips, Toth, and dual site Langmuir isotherms. The results for these models to represent the adsorption isotherms of $\mathrm{N}_{2} \mathrm{O}$ on BC-60 are presented in Figure 1. The constants obtained from the fitting these isotherms are reported in Tables 3-8. The results indicate that the best representation of the experimental adsorption data is observed using adsorption models that take into account heterogeneity of the energetic surface, which is not surprising since $\mathrm{BC}$ and $\mathrm{AC}$ are highly heterogeneous. These isotherm models correspond to the Sips, Toth, and dual-site Langmuir isotherms (cf. Tables 6-8), being the Toth isotherm model the one that exhibits the smallest fitting error likely for all temperatures due to the accurate representation of the low-pressure adsorption regime by this model.

The results for isosteric heats of adsorption $Q_{\mathrm{st}}$ for $\mathrm{N}_{2} \mathrm{O}$ on BC-60 are shown in Figure 2. These results were obtained in two different ways: (a) from direct numerical differentiation of isotherms at six different temperatures calculated using the parameters reported in Tables 1-8, and (b) from the equations derived in this work using the temperature dependence of the adjustable parameters, also reported in Tables 18. The equations presented in this work give values of $Q_{\text {st }}$ consistent with those obtained from numerical differentiation suggesting that small deviations are due to errors in fitting different parameters rather than in the derivation of the equations. It is interesting to note, that all the models converge to similar values of $Q_{\text {st }}$ only in the region where no extrapolation of the experimental data, corresponding to either low or high loadings) is needed. This region is represented by the blue rectangle shown in Figures 1-2. This behaviour highlights the uncertainty in the estimation of the zero coverage heats of adsorption from differentiation of isotherms at different temperatures, as well as the extrapolation to high loadings. Despite the errors in the fitting of the isotherms being small in all models, extrapolations to zero coverage predict values of the isosteric heat that may vary significantly based on the model selected. In this case, the Langmuir model and the second-order virial equation with 3 adjustable parameters exhibit similar behaviour in extrapolations at zero coverage, whereas the Toth isotherm and the first-order virial equation with two adjustable parameters give similar values at zero coverage. It should be highlighted that the $Q_{\text {st }}$ from the dual-Langmuir model have an unphysical maxima at low coverage, and that for both the Freundlich and Sips models $Q_{\text {st }}$ diverged at low coverages.

All the isotherm models previously discussed have in common that the adsorption equilibrium is described as the balance between the energetic contribution due to molecules adsorbed in active sites and the gain in entropy from the molecules escaping from the substrate. An alternative interpretation to the adsorption of fluids on solid substrates corresponds to the equilibrium of a 2D fluid phase, representing the adsorbed phase, with a 3D bulk phase. In this context, the so-called 2D-SAFT-VR equation of state has been previously reported to describe the adsorption of gases on solids. Here, this molecular-based equation of state has been used to represent the adsorption of $\mathrm{N}_{2} \mathrm{O}$ on BC-60. The SW parameters in the the 2D-SAFT-VR equation for bulk $\mathrm{N}_{2} \mathrm{O}$ gas $\left(\epsilon_{\text {bulk }}, \lambda_{\text {bulk }}\right.$ and $\sigma$ ) have been obtained from fitting to the critical values of density, temperature and pressure, according to the data reported by NIST (Lemmon et al., 2019). The SW bulk parameters are very similar to those used by other authors with similar molecular-based equations of state (Pereira et al., 2013). The adsorbed phase parameters have been obtained following the procedure used in previous 2D-SAFT-VR studies (Jiménez-Serratos et al., 2008; Trejos et al., 2014), assuming that the size of the particles and the energy-well depth of the SW interaction are not modified by the adsorption process, i.e., $\sigma_{\mathrm{ads}}=\sigma_{\mathrm{bulk}}$ and $\epsilon_{\mathrm{ads}}=\epsilon_{\mathrm{bulk}}$, respectively. The effect of the substrate on the adsorbed fluid is only taken into account in the range of the SW potential of the adsorbed molecules, $\lambda_{\text {ads }}$, that is different to the corresponding value in the bulk phase, and obtained by fixing the ratio of the critical temperature $R_{\mathrm{c}}=T_{c}^{\mathrm{ads}} / T_{c}^{\mathrm{bulk}}=0.4$. Since the low-limit of the isosteric heat is

$$
Q_{\mathrm{st}}=N_{0} \epsilon_{\mathrm{w}}+R T
$$


Table 9: Molecular parameters for the 2D-SAFT-VR equation of state for the adsorption of $\mathrm{N}_{2} \mathrm{O}$ on BC-60.

\begin{tabular}{lr}
\hline Parameter & \\
\hline$\lambda_{\text {bulk }}$ & 1.769 \\
$\sigma / \AA$ & 3.3551 \\
$\left(\epsilon_{\text {bulk }} / k\right) / \mathrm{K}$ & 161.01 \\
$\lambda_{\text {ads }}$ & 1.270 \\
$\sigma_{\text {ads }} / \AA$ & 3.3551 \\
$\left(\epsilon_{\text {ads }} / k\right) / \mathrm{K}$ & 161.01 \\
$\lambda_{\mathrm{w}}$ & 0.11 \\
$\left(\epsilon_{\mathrm{w}} / k\right) / \mathrm{K}$ & 2986.74 \\
$S_{0} /\left(\mathrm{m}^{2} \mathrm{~g}^{-1}\right)$ & 167.17 \\
$\omega /\left(\mathrm{K}^{-1}\right)$ & 0.002972 \\
\hline
\end{tabular}

the particle-surface SW energy $\epsilon_{w}$ was taken to reproduce $Q_{\mathrm{st}}$ in this limit, according to the experimental value at a temperature of $293.15 \mathrm{~K}$ (see Table 3 in reference (Cornelissen et al., 2013)). The remaining parameters, the range of the surface-particles attractive interaction $\lambda_{\mathrm{w}}$, that also gives an estimation of the size of the adsorbed layer, and the surface area $S$ were fitted to reproduce all the selected adsorption isotherms. The surface area was also fitted and reproduced according to the expression

$$
S=S_{0} e^{-\omega(T-263.15)},
$$

where $S_{0}$ corresponds to the surface area at $T=263.15 \mathrm{~K}$, and $\omega$ is a constant for all the isotherms. This equation is similar to Equation 13, that describes the temperature dependence of the saturation capacity in the Langmuir model. The isosteric heat predicted by the 2D-SAFT-VR EoS is similar to the predicted by the other adsorption isotherm models, and the prediction of this property at low coverages exhibits a similar behaviour as the one observed by the Langmuir and second-order virial models. 


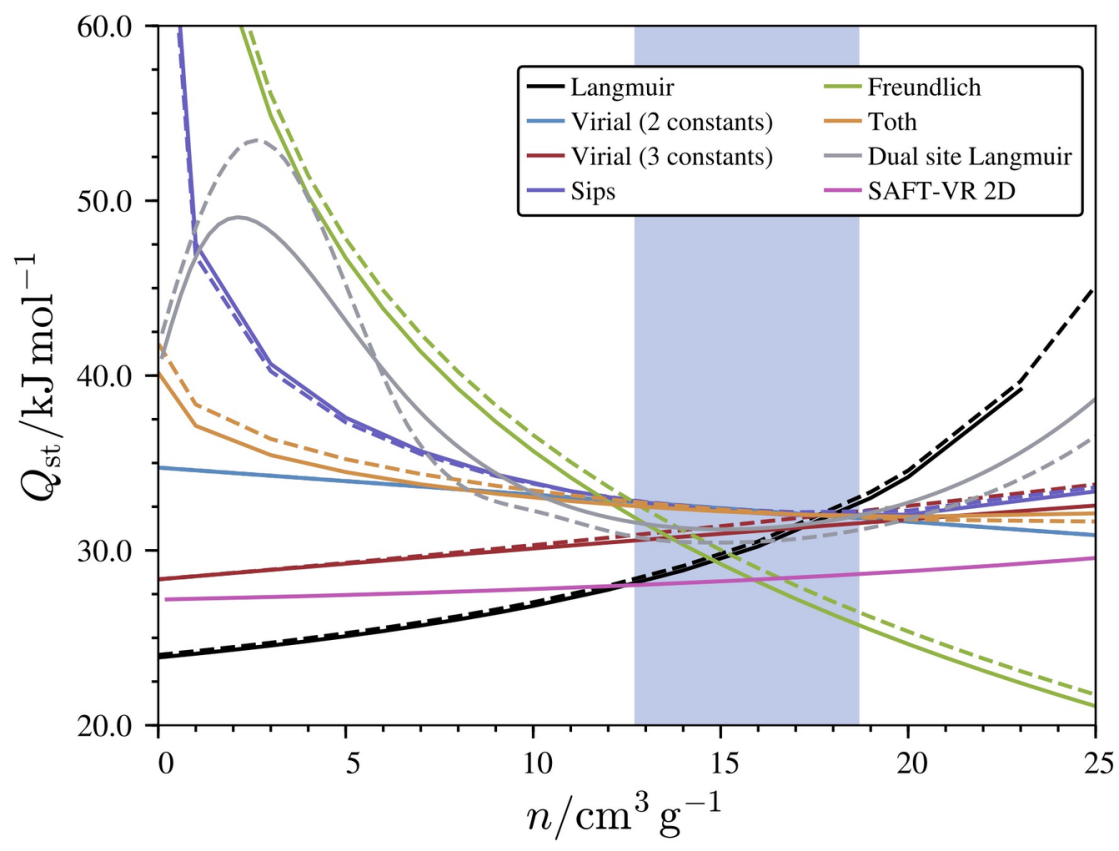

Figure 2: Isosteric heat of adsorption for $\mathrm{N}_{2} \mathrm{O}$ on biochar BC-60 based on experimental data from Cornelissen et al. (Cornelissen et al., 2013). The amount adsorbed $n$ is expressed as volume of $\mathrm{N}_{2} \mathrm{O}$ at STP per gram of sorbent, i.e., in units of $\mathrm{cm}^{3} \mathrm{~g}^{-1}$. Solid lines represent the isosteric heat of adsorption calculated from numerically differentiation of the isotherms reported at six different temperatures, while dashed lines are the results obtained from the equations derived in this work. Parameters for these models are in Tables 2-9. The blue area indicates the region where at least one experimental point for each experimental isotherm is present (cf. Figure 1). In this region, no extrapolation is needed to calculate the isosteric heats of adsorption.

\section{Conclusions}

In this work, we have presented a collection of equations to calculate the isosteric heat of adsorption for gases derived from some of the most common models for adsorption. The use of simple isotherm models is very common in the literature, despite of having evidence that the experimental data does not follow the assumptions of the selected model. More complex isotherms can adjust better experimental data, but often require more parameters to fit, as has been reviewed by Foo and Hameed (Foo \& Hameed, 2010). The use of a more complex approach like the 2D-SAFT-VR equation of state becomes attractive because less adjustable parameters are required than the simpler isotherm models, and also because it is possible to improve the molecular description through a more realistic intermolecular interaction, as recently reported using a Mie intermolecular potential model (Campos-Villalobos et al., 2019).

It is impossible to say which model discussed in this work is better or more accurate to extrapolate the isosteric heats of adsorption, but this paper highlights that care must be taken in the extrapolations of the heats of adsorption, even if the model fits well the adsorption isotherms. As our results demonstrate very different behaviour, even some unrealistic behaviour, is observed for both low and high coverage since these regions have been extrapolated. 


\section{Acknowledgments}

We are grateful for the support of the Royal Academy of Engineering Newton Research Collaboration Programme (3) - NRCP1516/1/124 for this work. Authors also acknowledge DAIP-University of Guanajuato (Mexico) for the grants CIIC 1143/2016 and CIIC 348/2019.

\section{Appendix A}

The 2D-SAFT-VR method (Martínez et al., 2007; Jiménez-Serratos et al., 2008) considers that molecules in both the adsorbed and bulk phases interact via SW potentials $u_{\mathrm{sw}}(r ; \sigma, \epsilon, \lambda)$,

$\begin{array}{ll} & \\ \infty & \text { if } r \leq \sigma \\ -\epsilon & \text { if } 0 \leq r \leq \lambda \sigma \\ 0 & \text { if } r>\lambda \sigma\end{array}$

$(41)$

where $\sigma$ is the particle's diameter, $\epsilon$ is the energy well-depth, and $\lambda$ is the attractive range of the potential. The model assumes that the bulk and adsorbed particles have the same size $\sigma$, but the attractive interaction is modified due to the effect of the substrate, and the values of the $\epsilon$ and $\lambda$ potential parameters corresponding to the bulk and adsorbed phases are different. We will denote by $\left(\epsilon_{\text {bulk }}, \lambda_{\text {bulk }}\right)$ and $\left(\epsilon_{\text {ads }}, \lambda_{\text {ads }}\right)$ the bulk and adsorbed SW particle-particle interactions parameters, respectively. Typically, $\epsilon_{\text {ads }} \approx 0.8 \epsilon_{\text {bulk }}$ and $\lambda_{\text {ads }}<\lambda_{\text {bulk }}$ in order to reproduce the experimental ratio of the critical temperatures of the adsorbed and bulk fluid phases $R_{c}=T_{c}^{\text {ads }} / T_{c}^{\text {bulk }} \approx 0.4$ (Sinanoglu \& Pitzer, 1960; Dash, 1975; Jiménez-Serratos et al., 2008). However, just in order to reduce the number of molecular parameters that requires the model, we can consider the effect of the substrate into the adsorbed particle-particle interaction only in the range of the potential and to assume that $\epsilon_{\mathrm{ads}}=\epsilon_{\mathrm{bulk}}$. This approximation has given accurate predictions of adsorption isotherms of CO2 in activated carbon with the Mie intermolecular potential (Campos-Villalobos et al., 2019) and we have used this approximation in this work.

The interaction potential $u_{\mathrm{pw}}$ exerted on the adsorbed particles by a planar structureless surface is also given by a SW potential with parameters $\left(\epsilon_{\mathrm{w}}, \lambda_{\mathrm{w}}\right)$, written as a function of the orthogonal coordinate distance $z$ between the particle and the surface. If $z=0$ is set at the position of contact of the particle with the surface then $u_{\mathrm{pw}}$ is given by:

$$
u_{\mathrm{pw}}(z)=\{
$$

$$
\begin{array}{ll}
\infty & \text { if } z<0 \\
-\epsilon_{\mathrm{w}} & \text { if } 0 \leq z \leq \lambda_{w} \sigma \\
0 & \text { if } z>\lambda_{\mathrm{w}} \sigma
\end{array}
$$


The Helmholtz free energy of the adsorbed fluid can be written as (Jiménez-Serratos et al., 2008; Trejos et al., 2014)

$$
\frac{A_{\mathrm{ads}}}{\eta_{\mathrm{ads}} R T}=\frac{A_{2 \mathrm{D}}}{\eta_{\mathrm{ads}} R T}-\ln \left(\frac{\lambda_{\mathrm{w}} \sigma}{\lambda_{\mathrm{B}}}\right)-N_{0} \epsilon_{\mathrm{w}} / R T
$$

where $\eta_{\text {ads }}$ is the number of moles in the adsorbed phase, $\lambda_{\mathrm{B}}$ is the de Broglie's thermal wavelength, and $N_{0}$ is Avogadro's number. Details of the Helmholtz free energy of the bulk fluid are given in (Jiménez-Serratos et al., 2008; Trejos et al., 2014).

The thermodynamic properties of the adsorbed phase are determined by the equilibrium condition

$$
\mu_{\mathrm{bulk}}=\mu_{\mathrm{ads}}
$$

where $\mu_{\text {bulk }}=\partial A_{\text {bulk }} / \partial \eta_{\text {bulk }}$ and $\mu_{\text {ads }}=\partial A_{\text {ads }} / \partial \eta_{\text {ads }}$ are the chemical potentials of the bulk and adsorbed phases, respectively. From this equation it is then possible to give the bulk pressure, $p$, at low values of $\eta_{\text {bulk }}$,

$$
p=\frac{R T \rho_{\mathrm{ads}}}{\lambda_{\mathrm{w}} \sigma} e^{\left[\Delta \mu_{2 D}-N_{0} \epsilon_{\mathrm{w}}\right] / R T}
$$

where $\rho_{\text {ads }}=\eta_{\text {ads }} / S$ and $\Delta \mu_{2 \mathrm{D}}$ are the molar density and excess chemical potential of the adsorbed fluid, respectively, and $S$ is the surface area. According to Equations (1) and (41), the isosteric heat of the 2D-SAFT-VR is given by

$$
Q_{\mathrm{st}}=N_{0} \epsilon_{\mathrm{w}}+R T+R T^{2} \frac{\partial}{\partial T}\left(\frac{\Delta \mu_{2 \mathrm{D}}}{R T}\right)
$$

For non-associating fluids, the first order-perturbation term for $\Delta \mu_{2 \mathrm{D}}$ is given by (Trejos et al., 2014):

where $\alpha_{\mathrm{vdW}}$ is the van der Waals energy constant, $\gamma$ is the $2 \mathrm{D}$ packing fraction $\gamma=N_{0} \rho_{\text {ads }} \pi \sigma^{2} / 4, g_{\mathrm{hd}}\left(\sigma, \gamma_{\mathrm{eff}}\right)$ is the contact value of the hard-disks radial distribution function given in terms on an effective packing 
fraction $\gamma_{\mathrm{eff}}$, and $\Phi\left(\gamma_{\mathrm{eff}}\right)$ is a function determined by $g_{\mathrm{hd}}$ and its first density derivative. Following previous work, we use the Henderson hard-disks equation of state (Henderson, 1975),

$$
g_{\text {hd }}\left(\sigma, \gamma_{\text {eff }}\right)=\frac{1-7 \gamma_{\text {eff }} / 16}{\left(1-\gamma_{\text {eff }}\right)^{2}}
$$

and

$$
\gamma_{\mathrm{eff}}=d_{1} \gamma+d_{2} \gamma^{2}
$$

with

$$
d_{1}=1.4215-0.405625 \lambda_{\mathrm{ads}}-0.03869981 \lambda_{\mathrm{ads}}^{2}
$$

$$
d_{2}=1.5582-1.89768 \lambda_{\mathrm{ads}}+0.405215 \lambda_{\mathrm{ads}}^{2}
$$

and

$$
\alpha_{\mathrm{vdW}}=\pi \epsilon_{\mathrm{ads}} \sigma^{2}\left(\lambda_{\mathrm{ads}}^{2}-1\right) / 2
$$

The corresponding expression for $\Phi\left(\gamma_{\text {eff }}\right)$ is

$$
\Phi=1+\frac{\left(25-7 \gamma_{\mathrm{eff}}\right)\left(2 \gamma_{\mathrm{eff}}-d_{1} \gamma\right)}{2\left(1-\gamma_{\mathrm{eff}}\right)\left(16-7 \gamma_{\mathrm{eff}}\right)}
$$

The expressions for the augmented vdW equation of state can be obtained from Eq.(??) assuming $g_{\text {hd }}\left(\sigma, \gamma_{\text {eff }}\right)=1$, and consequently $\Phi\left(\gamma_{\text {eff }}\right)=1$. 


\section{References}

Nicholson, D.; Gubbins, K. E. Separation of carbon dioxide-methane mixtures by adsorption: Effects of geometry and energetics on selectivity. J. Chem. Phys. 1996, 104, 8126-8134

Balbuena, P. B.; Gubbins, K. E. Theoretical interpretation of adsorption behavior of simple fluids in slit pores. Langmuir 1993, 9 , 1801-1814

Snurr, R. Q.; Bell, A. T.; Theodorou, D. N. Prediction of adsorption of aromatic hydrocarbons in silicalite from grand canonical Monte Carlo simulations with biased insertions. J. Phys. Chem. 1993 , 97 , 1374213752

Sircar, S.; Cao, D. Heat of Adsorption. Chem. Eng. Technol. 2002 , 25 , 945-948

Hill, T. L. Statistical Mechanics of Adsorption. V. Thermodynamics and Heat of Adsorption. J. Chem. Phys. $1949,17,520-535$

Myers, A. L. Thermodynamics of adsorption in porous materials. AIChE J. 2002 , 48, 145-160

Builes, S.; Sandler, S. I.; Xiong, R. Isosteric Heats of Gas and Liquid Adsorption. Langmuir 2013 , 29 , 10416-10422

Langmuir, I. The constitution and fundamental properties of solids and liquids. Part I. Solids. J. Am. Chem Soc. 1916 , $38,2221-2295$

Honig, J. M.; Mueller, C. R. Adaptation of lattice vacancy theory to gas adsorption phenomena. J. Phys. Chem. 1962, 66, 1305-1308

Nitta, T.; Shigetomi, T.; Kuro-Oka, M.; Katayama, T. An adsorption isotherm of multi-site occupancy model for homogeneous surface. J. Chem. Eng. Jpn. 1984, 17, 39-45

Martinez, G. M.; Basmadjian, D. Towards a general gas adsorption isotherm. Chem. Eng. Sci. 1996 , 51 , $1043-1054$

Yao, C. Extended and improved Langmuir equation for correlating adsorption equilibrium data. Sep. Purif. Technol. 2000 , 19 , 237-242

Bai, R.; Yang, R. T. A Thermodynamically Consistent Langmuir Model for Mixed Gas Adsorption. J. Colloid Interface Sci $\mathbf{2 0 0 1}$, 239, 296-302

Zhang, P.; Wang, L. Extended Langmuir equation for correlating multilayer adsorption equilibrium data. Separation and Purification Technology 2010 , 70 , 367-371

Czepirski, L.; Jagiello, J. Virial-type thermal equation of gas-solid adsorption. Chem. Eng. Sci. 1989 , 44 , $797-801$

Siperstein, F. R.; Myers, A. L. Mixed-gas adsorption. AIChE J. 2001 , 47 , 1141-1159

Freundlich, H. M. F. Über die adsorption in lösungen. Z. Phys. Chem. 1906 , 57, 385-471

Sips, R. On the Structure of a Catalyst Surface. J. Chem. Phys. 1948 , 16 , 490-495

Toth, J. State equations of the solid gas interface layer. Acta Chem. Acad. Hung 1971 , 69, 311-317

Gil-Villegas, A.; Galindo, A.; Whitehead, P. J.; Mills, S. J.; Jackson, G.; Burgess, A. N. Statistical Associating Fluid Theory for Chain Molecules with Attractive Potentials of Variable Range. J. Chem. Phys. 1997 , $106,4168-4186$

Martínez, A.; Castro, M.; McCabe, C.; Gil-Villegas, A. Predicting adsorption isotherms using a twodimensional statistical associating fluid theory. J. Chem. Phys. 2007 , 126, 074707 
Jiménez-Serratos, G.; Santillán, S.; Avendaño, C.; Castro, M.; Gil-Villegas, A. Molecular Thermodynamics of Adsorption using Discrete-Potential Systems. Oil \& Gas Sci. Tech. 2008 , 63 , 329-341

Trejos, V. M.; Becerra, M.; Figueroa-Gerstenmaier, S.; Gil-Villegas, A. Theoretical modelling of adsorption of hydrogen onto graphene, MOF's and other carbon-based substrates. Mol. Phys. 2014, 112 , 2330-2338

Lee, L. L. Molecular Thermodynamics of Nonideal Fluids ; Butterworths Series in Chemical Engineering: Stoneham, MA, USA, 1988

Mason, J. A.; Sumida, K.; Herm, Z. R.; Krishna, R.; Long, J. R. Evaluating metal-organic frameworks for post-combustion carbon dioxide capture via temperature swing adsorption. Energy Environ. Sci. 2011 , $4,3030-3040$

Brunauer, S.; Emmett, P. H.; Teller, E. Adsorption of Gases in Multimolecular Layers. J. Am. Chem Soc. $1938,60,309-319$

Dill, K. A.; Bromberg, S. In Molecular Driving Forces. Statistical Thermodynamics in Biology, Chemistry, Physics, and Nanoscience ; Second,, Ed.; Garland Science: New York, 2010

Machin, W. D.; Ross, S. On Physical Adsorption. XVII. Experimental Verification of the Two-Dimensional van der Waals Equation of State Above and Below the Critical Temperature. Proc. R. Soc. Lond. A 1962 , $265,455-462$

Dash, J. G. Films on Solid Surfaces ; Academic Press: New York, 1975

Barker, J.; Henderson, D. Perturbation theory and equation of state for fluids: the square-well potential. $J$. Chem. Phys. 1967, 47, 2856-2861

del Río, F.; Gil-Villegas, A. Monolayer Adsorption of the Square-Well fluid of Variable Range. J. Phys. Chem. 1991, 95, 787-792

Chapman, W. G.; Jackson, G.; Gubbins, K. E. Phase equilibria of associating fluids. Mol. Phys. 1988 , 65 , 1057-1079

Jackson, G.; Chapman, W. G.; Gubbins, K. E. Phase equilibria of associating fluids. Mol. Phys. 1988 , 65 , $1-31$

Chapman, W. G.; Gubbins, K. E.; Jackson, G.; Radosz, M. SAFT: Equation of state solution model for associating fluids. Fluid Phase Equil. 1989 , 52 , 31-38

Chapman, W. G.; Gubbins, K. E.; Jackson, G.; Radosz, M. New reference equation of state for associating liquids. Ind. Eng. Chem. Res. 1990 , 29 , 1709-1721

Galindo, A.; Davies, L. A.; Gil-Villegas, A.; Jackson, G. The Thermodynamics of Mixtures and the Corresponding Mixing Rules in the SAFT-VR approach for Potentials of Variable Range. Mol. Phys. 1998 , 93 , 241-252

Castro, M.; Martínez, A.; Gil-Villegas, A. Modelling Adsorption Isotherms of Binary Mixtures of Carbon Dioxide, Methane and Nitrogen. Adsorpt. Sci. Technol. 2011, 29, 59-70

Martínez, A.; Trejos, V. M.; Gil-Villegas, A. Predicting adsorption isotherms for methanol and water onto different surfaces using the SAFT-VR-2D approach and molecular simulation. Fluid Phase Equilib. 2017 , 449, 207-216

Castro, M.; Mendoza, J. L.; Buenrostro-González, E.; López, S.; Gil-Villegas, A. Predicting adsorption isotherms of asphaltenes in porous materials. Fluid Phase Equil. 2009 , 286 , 113-119

Cornelissen, G.; Rutherford, D. W.; Arp, H. P. H.; Dorsch, P.; Kelly, C. N.; Rostad, C. E. Sorption of Pure $\mathrm{N}_{2} \mathrm{O}$ to Biochars and Other Organic and Inorganic Materials under Anhydrous Conditions. Environ. Sci. Technol. 2013 , 47, 7704-7712 
Li, H.; Dong, X.; da Silva, E. B.; de Oliveira, L. M.; Chen, Y.; Ma, L. Q. Mechanisms of metal sorption by biochars: Biochar characteristics and modifications. Chemosphere 2017 , 178, 466-478

Lehmann, J. Bio-energy in the black. Front. Ecol. Environ. 2007 , 5 , 381-387

Thomson, A. J.; Giannopoulos, G.; Pretty, J.; Baggs, E. M.; Richardson, D. J. Biological sources and sinks of nitrous oxide and strategies to mitigate emissions. Phil. Trans. R. Soc. B 2012, 367, 1157-1168

Portmann, R. W.; Daniel, J. S.; Ravishankara, A. R. Stratospheric ozone depletion due to nitrous oxide: influences of other gases. Phil. Trans. R. Soc. B 2012, 367, 1256-1264

Lemmon, E. W.; McLinden, M. O.; Friend, D. G. Thermophysical Properties of Fluid Systems, NIST Chemistry WebBook ; NIST Standard Reference Database Number 69, Eds. P. J. Linstrom and W. G. Mallard, 2019

Pereira, L. M. C.; Oliveira, M. B.; Dias, A. M. A.; Llovel, F.; Vega, L. F.; Carvalho, P. J.; Coutinho, J. A. P. High pressure separation of greenhouse gases from air with 1-ethyl-3- methylmidazolium methylphosphonate. Int. J. Green. Gas Control 2013 , 19, 299-309

Foo, K. Y.; Hameed, B. H. Insights into the modeling of adsorption isotherm systems. Chem. Eng. J. 2010 $, 156,2-10$

Campos-Villalobos, G.; Ravipati, S.; Haslam, A. J.; Jackson, G.; Suaste, J.; Gil-Villegas, A. Modelling adsorption using an augmented two-dimensional statistical associating fluid theory: 2D-SAFT-VR Mie. Mol. Phys. 2019 , 117 , 3770-3782

Sinanoglu, O.; Pitzer, K. S. Interactions between Molecules Adsorbed on a Surface. J. Chem. Phys. 1960 , 32, $1279-1288$

Henderson, D. A simple equation of state for hard discs. Mol. Phys. 1975 , 30 , 971-972

\section{References}

Separation of carbon dioxide-methane mixtures by adsorption: Effects of geometry and energetics on selectivity. (1996). J. Chem. Phys., 104, 8126-8134. https://doi.org/10.1063/1.471527

Theoretical interpretation of adsorption behavior of simple fluids in slit pores. (1993). Langmuir, 9, 1801-1814. https://doi.org/10.1021/la00031a031

Prediction of adsorption of aromatic hydrocarbons in silicalite from grand canonical Monte Carlo simulations with biased insertions. (1993). J. Phys. Chem., 97, 13742-13752. https://doi.org/10.1021/j100153a051

Heat of Adsorption. (2002). Chem. Eng. Technol., 25, 945-948. https://doi.org/10.1002/15214125 (20021008) 25:10<945: :AID-CEAT945>3.0.CO;2-F

Statistical Mechanics of Adsorption. V. Thermodynamics and Heat of Adsorption. (1949). J. Chem. Phys., 17, 520-535. https://doi.org/https://doi.org/10.1063/1.1747314

Thermodynamics of adsorption in porous materials. (2002). AIChE J., 48, 145-160. https://doi.org/ https://doi.org/10.1002/aic.690480115

Isosteric Heats of Gas and Liquid Adsorption. (2013). Langmuir, 29, 10416-10422. https://doi.org/ https://doi.org/10.1021/la401035p

The constitution and fundamental properties of solids and liquids. Part I. Solids.. (1916). J. Am. Chem Soc., 38, 2221-2295. https://doi.org/https://doi.org/10.1021/ja02268a002

Adaptation of lattice vacancy theory to gas adsorption phenomena. (1962). J. Phys. Chem., 66, 1305-1308. https://doi.org/https://doi.org/10.1021/j100813a022 
An adsorption isotherm of multi-site occupancy model for homogeneous surface. (1984). J. Chem. Eng. Jpn., 17, 39-45. https://doi.org/https://doi.org/10.1252/jcej.17.39

Towards a general gas adsorption isotherm. (1996). Chem. Eng. Sci., 51, 1043-1054. https://doi.org/ https://doi.org/10.1016/S0009-2509(96)80004-2

Extended and improved Langmuir equation for correlating adsorption equilibrium data. (2000). Sep. Purif. Technol., 19, 237-242. https://doi.org/https://doi.org/10.1016/S1383-5866(00)00060-5

A Thermodynamically Consistent Langmuir Model for Mixed Gas Adsorption. (2001). J. Colloid Interface Sci, 239, 296-302. https://doi.org/https://doi.org/10.1006/jcis.2001.7563

Extended Langmuir equation for correlating multilayer adsorption equilibrium data. (2010). Separation and Purification Technology, 70, 367-371. https://doi.org/https://doi.org/10.1016/j.seppur.2009.10. 007

Virial-type thermal equation of gas-solid adsorption. (1989). Chem. Eng. Sci., 44, 797-801. https: //doi.org/https://doi.org/10.1016/0009-2509(89)85253-4

Mixed-gas adsorption. (2001). AIChE J., 47, 1141-1159. https://doi.org/https://doi.org/10.1002/ aic. 690470520

Über die adsorption in lösungen. (1906). Z. Phys. Chem., 57, 385-471. https://doi.org/https://doi. org/10.1515/zpch-1907-5723

On the Structure of a Catalyst Surface. (1948). J. Chem. Phys., 16, 490-495. https://doi.org/https: //doi.org/10.1063/1.1746922

State equations of the solid gas interface layer. (1971). Acta Chem. Acad. Hung, 69, 311-317.

Statistical Associating Fluid Theory for Chain Molecules with Attractive Potentials of Variable Range. (1997). J. Chem. Phys., 106, 4168-4186. https://doi.org/https://doi.org/10.1063/1.473101

Predicting adsorption isotherms using a two-dimensional statistical associating fluid theory. (2007). J. Chem. Phys., 126, 074707. https://doi.org/https://doi.org/10.1063/1.2483505

Molecular Thermodynamics of Adsorption using Discrete-Potential Systems. (2008). Oil \& Gas Sci. Tech., 63, 329-341. https://doi.org/https://doi.org/10.2516/ogst:2008027

Theoretical modelling of adsorption of hydrogen onto graphene, MOF's and other carbon-based substrates. (2014). Mol. Phys., 112, 2330-2338. https://doi.org/https://doi.org/10.1080/00268976.2014. 903591

Molecular Thermodynamics of Nonideal Fluids. (1988). Butterworths Series in Chemical Engineering. https://doi.org/https://doi.org/10.1016/C2013-0-01069-9

Adsorption of Gases in Multimolecular Layers. (1938). J. Am. Chem Soc., 60, 309-319. https://doi.org/ http://dx.doi.org/10.1021/ja01269a023

Second (Ed.). (2010). Molecular Driving Forces. Statistical Thermodynamics in Biology, Chemistry, Physics, and Nanoscience. Garland Science.

On Physical Adsorption. XVII. Experimental Verification of the Two-Dimensional van der Waals Equation of State Above and Below the Critical Temperature. (1962). Proc. R. Soc. Lond. A, 265, 455-462. https://doi.org/https://doi.org/10.1098/rspa.1962.0035

Films on Solid Surfaces. (1975). Academic Press. https://doi.org/https://doi.org/10.1016/B978-012-203350-6.X5001-4

Perturbation theory and equation of state for fluids: the square-well potential. (1967). J. Chem. Phys., 47(3), 2856-2861. https://doi.org/http://dx.doi.org/10.1063/1.1712308 
Monolayer Adsorption of the Square-Well fluid of Variable Range. (1991). J. Phys. Chem., 95, 787-792. https://doi.org/https://doi.org/10.1021/j100155a056

Phase equilibria of associating fluids. (1988). Mol. Phys., 65, 1057-1079. https://doi.org/10.1080/ 00268978800101601

Phase equilibria of associating fluids. (1988). Mol. Phys., 65, 1-31. https://doi.org/10.1080/ 00268978800100821

SAFT: Equation of state solution model for associating fluids. (1989). Fluid Phase Equil., 52, 31-38. https://doi.org/10.1016/0378-3812(89)80308-5

New reference equation of state for associating liquids. (1990). Ind. Eng. Chem. Res., 29, 1709-1721. https://doi.org/10.1021/ie00104a021

The Thermodynamics of Mixtures and the Corresponding Mixing Rules in the SAFT-VR approach for Potentials of Variable Range. (1998). Mol. Phys., 93, 241-252. https://doi.org/https://doi.org/10. 1080/002689798169249

Modelling Adsorption Isotherms of Binary Mixtures of Carbon Dioxide, Methane and Nitrogen. (2011). Adsorpt. Sci. Technol., 29, 59-70. https://doi.org/https://doi.org/10.1260/0263-6174.29.1.59

Predicting adsorption isotherms for methanol and water onto different surfaces using the SAFT-VR-2D approach and molecular simulation.. (2017). Fluid Phase Equilib., 449, 207-216. https://doi.org/https: //doi.org/10.1016/j.fluid.2017.06.025

Predicting adsorption isotherms of asphaltenes in porous materials. (2009). Fluid Phase Equil., 286, 113-119. https://doi.org/https://doi.org/10.1016/j.fluid.2009.08.009

Sorption of Pure $\mathrm{N}_{2} \mathrm{O}$ to Biochars and Other Organic and Inorganic Materials under Anhydrous Conditions. (2013). Environ. Sci. Technol., 47, 7704-7712. https://doi.org/https://doi.org/10.1021/es400676q

Mechanisms of metal sorption by biochars: Biochar characteristics and modifications. (2017). Chemosphere, 178, 466-478. https://doi.org/https://doi.org/10.1016/j.chemosphere.2017.03.072

Bio-energy in the black. (2007). Front. Ecol. Environ., 5, 381-387. https://doi.org/10.1890/15409295(2007)5[381:BITB] 2.0.CD;2

Biological sources and sinks of nitrous oxide and strategies to mitigate emissions. (2012). Phil. Trans. R. Soc. B, 367, 1157-1168. https://doi.org/10.1098/rstb.2011.0415

Stratospheric ozone depletion due to nitrous oxide: influences of other gases. (2012). Phil. Trans. R. Soc. B, 367, 1256-1264. https://doi.org/10.1098/rstb.2011.0377

Thermophysical Properties of Fluid Systems, NIST Chemistry WebBook. (2019). NIST Standard Reference Database Number 69, Eds. P. J. Linstrom and W. G. Mallard. https://doi.org/10.18434/T4D303

High pressure separation of greenhouse gases from air with 1-ethyl-3- methylmidazolium methyl-phosphonate. (2013). Int. J. Green. Gas Control, 19, 299-309. https://doi.org/https://doi.org/10.1016/j.ijggc . 2013.09.007

Insights into the modeling of adsorption isotherm systems. (2010). Chem. Eng. J., 156, 2-10. https: //doi.org/https://doi.org/10.1016/j.cej.2009.09.013

Modelling adsorption using an augmented two-dimensional statistical associating fluid theory: 2D-SAFT-VR Mie. (2019). Mol. Phys., 117, 3770-3782. https://doi.org/https://doi.org/10.1080/00268976.2019. 1665724

Interactions between Molecules Adsorbed on a Surface. (1960). J. Chem. Phys., 32, 1279-1288. https: //doi.org/https://doi.org/10.1063/1.1730910 
A simple equation of state for hard discs. (1975). Mol. Phys., 30, 971-972. https://doi.org/https: //doi.org/10.1080/00268977500102511 